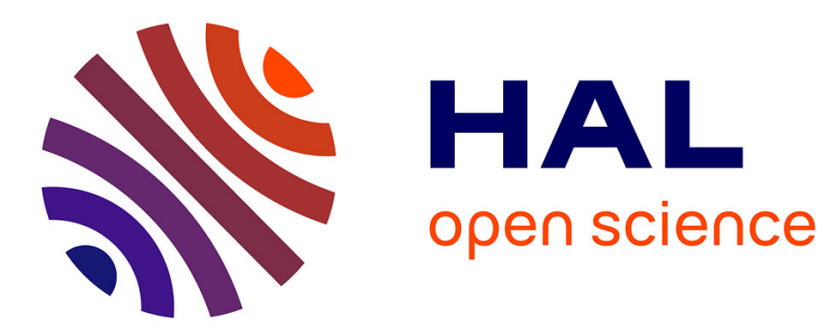

\title{
SUR LA RECHERCHE DU BACTERIUM COLI DANS LE LAIT
}

Jean Pien, Jacques Bachimont

\section{To cite this version:}

Jean Pien, Jacques Bachimont. SUR LA RECHERCHE DU BACTERIUM COLI DANS LE LAIT. Le Lait, 1932, 12 (119), pp.903-914. hal-00895080

\section{HAL Id: hal-00895080 \\ https://hal.science/hal-00895080}

Submitted on 1 Jan 1932

HAL is a multi-disciplinary open access archive for the deposit and dissemination of scientific research documents, whether they are published or not. The documents may come from teaching and research institutions in France or abroad, or from public or private research centers.
L'archive ouverte pluridisciplinaire HAL, est destinée au dépôt et à la diffusion de documents scientifiques de niveau recherche, publiés ou non, émanant des établissements d'enseignement et de recherche français ou étrangers, des laboratoires publics ou privés. 
de resserrements. Les conidies sont elliptiques, $4 \times 2,6 \mu$, d'abord hyalines, plus tard roses et saupoudrant d'une façon très dense le mycelium. Sur purée de pommes de terre en tube, on observe une bonne croissance de mycelium blanc. L'examen des zones brunes sur la surface inférieure de la croissance montre la présence de cellules mycéliales enflées et de macrospores semblables à celles de HORNe et WILLIAMson pour l'Eidamia catenulata.

Dans les cultures sur lait, il y a eu croissance feutrée sur la surface du milieu et les côtés du tube, tandis que la surface inférieure de la croissance était de couleur jaune ; un caillé tendre était formé et lentement solubilisé. Pas de modification de couleur dans le lait additionné de carbonate de chaux, mais dans le lait-tournesol, une réaction acide fut visible.

Après ensemencement dans une solution de CzAPEK et chauffage à $70^{\circ} \mathrm{C}$. dans un bain-marie, pas de développement du champignon. Toutefois, après chauffage à $60^{\circ} \mathrm{C}$., il y a eu croissance. Conservé à $8^{\circ} \mathrm{C}$. pendant 3 semaines en magasin frigorifique, dont l'atmosphère était maintenue humide par la présenee de saumure en cuves ouvertes, la croissance fut normale; mais conservé à $5^{\circ} \mathrm{C}$. pendant 3 semaines, pas d'augmentation apparente dans les dimensions de la colonie.

Sur solution de CzapeK eontenant $10 \%$ de $\mathrm{NaCl}$, eroissance, mais pas de croissance quand la solution de Czapek contenait $15 \%$ de NaCl. Quand il y avait $5 \%$ de $\mathrm{NaCl}$ dans la solution de CzAPEK, eroissance visible sous la forme d'un voile blanchâtre, gélatineux et submergé, avec quelques morceaux en surface, minces, éparpillés, blanchâtres. L'hyphe devint très cloisonné et se rompit en spores arrondies, donnant une apparence analogue à l'Oidium. Ces spores ne furent pas observées dans les solutions plus concentrées en $\mathrm{NaCl}$.

Dans une culture en solution de CzApEK, après 4 semaines, $p H$ du milieu $=6,4 ; p \mathrm{H}$ originel du milieu $=7,5$.

Très bonne eroissance sur gélatine, donnant des corémies ramifiées, analogues à des doigts. La gélatine fut partiellement $(50 \%)$ liquéfiée en 8 jours, aveo de petites bulles de gaz dans la partie non liquéfiée, et la réaction était acide $(p \mathrm{H}=5)$

Production de diastase. - Bonne.

(A suivre.)

\section{SUR LA RECHERCHE DU BACTERIUM COLI DANS LE LAIT}

\section{JWAN PIEN}

Ingénieur chimiste (I. C. R.)

Docteur ès Sciences

Directeur des Laboratoires

des "Fermiers Réunis" par

et JACQUES BACHIMONT

du Laboratoire de Bactériologie des "Fermiers Réunis"

\section{PREMIER ARTICLE.}

Avant de procéder à cette étude nous croyons intéressant :

10 De donner une idée du nombre de colibacilles que l'on rencontre ordinairement dans les laits ; 
$2^{\circ}$ D'indiquer les raisons de l'intérêt qui s'attache à la recherche de ce microbe ;

$3^{\circ}$ D'essayer de fixer l'ordre de grandeur de la sensibilité qu'on peut exiger d'une méthode de recherche.

\section{§ 1. - TENEUR APPROXIMATIVE DES LAITS EN COLIBACILLES,}

Une distinction s'impose immédiatement suivant que l'on considère les laits crus ou les laits pasteurisés.

$1^{\circ}$ Dans le lait cru la présence du $B$. Coli est un fait constant, régulier et à peu près inévitable avec les techniques actuelles de récolte du lait dans les étables. Il n'est pas nécessaire d'insister sur le fait que presque tous les éléments d'une étable, si propre soit-elle (litières, sol, atmosphère, animaux), constituent des milieux très riches en germes variés, au nombre desquels le $B$. Coli figure en bonne place. Il est done impossible, même en utilisant la traite mécanique (l'expérience l'a montré), de ne pas introduire du colibacille dans le lait, si peu que ce soit.

Les doses varient considérablement. Mais en général elles sont, d'après nos recherches personnelles dans ce domaine, toujours supérieures, et de beaucoup bien souvent, à 1.000 individus au litre de-lait au sortir de l'étable. Dans les fermes particulièrement bien soignées (animaux et personnel très propres) on arrive, selon nous, à se tenir à des doses de coli comprises entre quelques centaines et quelques milliers au litre. Dans les fermes courantes, où, comme on le sait, les plus élémentaires précautions sont négligées, nous avons trouvé des teneurs de l'ordre de 100.000 à plusieurs millions. Les chiffres publiés récemment par VASSILEFF [1] confirment cette donnée.

$2^{\circ}$ Dans le lait pasteurisé la question, bien entendu, est toute différente. Dans une pasteurisation très bien faite, en vase clos (à condition de se tenir, pour la pasteurisation basse, à $65-66^{\circ}$ pendant 30 minutes), on ne retrouve pas de coli dans le lait pris aseptiquement au sortir des appareils. De nombreux travaux américains l'ont surabondamment prouvé, en particulier ceux de AYERS et Johnson [2], Tanner et Dubois Granville [3], Johnson [4], Tanner et WINDSOR [5]. Nos recherches nous ont confirmé dans cette opinion et nous ont appris également que la température de pasteurisation basse ordinairement fixée à $63^{\circ}$ est souvent insuffisantē, et qu'il faut se tenir aux environs de $65^{\circ}$ pour ne pas retrouver de $B$. Coli, d'une manière régulière, dans le lait.

Nous croyons maintenant devoir insister sur le fait suivant, que la pratique nous a enseigné : quoique la grande majorité des laits bien pasteurisés soient exempts de colibacilles au sortir même du pasteurisateur, les divers traitements que subit le lait avant de 
parvenir au consommateur (refroidissement, mise en pots, transport, divisionnement à la vente au détail) sont, bien souvent, autant de eauses de réinfection, d'ailleurs difficilement évitables, qui font que le lait du commerce est fréquemment chargé en germes de toutes sortes et de colibacilles en particulier. VASSILEFF donne, pour les laits pasteurisés du commerce, des teneurs comprises entre 20.000 et 100.000 au litre.

Si on supprime les eauses de réinfection que nous venons de signaler en pasteurisant et réfrigérant à l'abri de l'air, en effectuant le remplissage aseptiquement (toujours en appareils clos) dans des récipients stériles, on arrive à ne pas retrouver de colibacilles dans le lait sur 10 et même sur $50 \mathrm{~cm}^{3}$ (ce qui correspond à moins de 20 colibacilles au litre). Ce fait, que nous avons l'occasion d'observer quotidiennement sur un lait entouré de soins exceptionnels (le lait $\mathrm{OFCO}$ ), nous confirme dans l'idée émise plus haut et dans la valeur des techniques qui seront exposées dans la suite de ce travail.

\section{§ 2. - INTÉRÊT DE LA RECHERCHE DU COLIBACILLE} DANS LE LAIT.

Cet intérêt est basé, selon nous, sur des raisons d'ordre hygiénique et industriel.

10 Raisons relevant de l'hygiène. - La question de l'impossibilité quasi absolue de. mettre en vente du lait exempt de $B$. Coli se rattache immédiatement à la suivante : La présence $d u$ colibacille dans le lait constitue-t-elle un danger? Les médecins répondent : Non, car le colibacille n'est pas nocif en soi. Bien que le bacille d'Escherich devienne parfois pathogène, tant pour l'homme que pour l'animal, il est le plus souvent réduit à l'état de simple saprophyte inoffensif. Sa signification dans le lait serait done assez différente de celle qu'il prend dans les eaux, car l'origine du colibacille des eaux serait, en général, plus suspecte que celle de ce même germe dans le lait. Ce raisonnement, qui tend à diminuer la signification et l'importance du coli dans le lait, n'est peut être pas, à notre avis, rigoureusement inattaquable. Il mériterait d'être étudié d'un peu près à la lumière des idées suivantes :

a) On sait que la signification du colibacille dans les eaux est en quelque sorte liée à la grave question de la présence du bacille typhique. Sur les raisons de ee lien, Dopter et Sacquépé e [6] s'expriment ainsi : «... Il est avéré que la recherche du bacille typhique dans les eaux est très difficile, pour ne pas dire illusoire, car les divers procédés d'isolement aboutissent à peu près uniquement à la découverte de colibacille, à l'exclusion du B. typhique, même dans les eaux notoirement typhogènes. D'autre part le typhique provient des excreta ou des cadavres ; on conçoit difficilement qu'ils puissent 
passer dans les eaux sans être accompagnés des microbes constamment présents dans les excreta et les cadavres; ces microbes (colibacille en particulier) constituent en quelque sorte, leurs satellites obligés. Parmi eux, et pour des raisons techniques, e'est encore le colibacille qu'il est plus facile de prendre comme témoin. "

DiénerT et ETRILlaRd [7] sont même allés plus loin. Il résulte, en effet, de leurs recherches, qu'on ne rencontre pratiquement jamais de bacille typhique dans une eau sans rencontrer en même temps du colibacille (l'inverse n'ayant pas lieu). De sorte que l'absence de colibacille correspondrait nécessairement à l'absence de typhique.

On en déduit logiquement que, plus une eau renferme de colibacilles plus elle a été infectée et plus elle a de chances de renfermer du typhique. C'est pourquoi tous les bactériologistes admettent qu'il faut dans les analyses d'eaux, non seulement constater la présence du coli, mais encore préciser son abondance. VINCENT estime $q u$ 'une teneur inférieure à 50 colibacilles au litre d'eau ne la rend pas suspecte, alors qu'elle le devient au-dessus de cette dose et qu'on doit la considérer comme mauvaise ou très mauvaise au-dessus de 1.000 au litre. La colimétrie est un élément d'appréciation de premier ordre de la qualité bactériologique des eaux.

b) Ce raisonnement vaut-il pour le lait? Bornons-nous à en discuter pour les laits pasteurisés. De tels laits sont (ou peuvent être) au sortir des appareils, complètement exempts des colibacilles qu'ils contenaient sûrement, et des typhiques qu'ils auraient pu éventuellement renfermer avant la pasteurisation. Ces laits, disions-nous plus haut, à de très rares exceptions près, vont être, au cours des manipulations et de la mise en vente, inévitablement souillés, quelles que soient l'attention et la bonne volonté des personnes chargées de ces opérations. La nature globale de ces souillures n'est pas exactement la même que celle des éléments qui contaminent les eaux. Les déjections humaines ou animales, les cadavres d'animaux. ne s'introduisent évidemment pas direotement dans le lait, comme c'est le cas pour les eaux. Mais les germes qui proviennent de ces sources d'infection peuvent être transportés accidentellement dans le lait par des récipients lavés avec des eaux contaminées, par les mains des ouvriers, par l'atmosphère des usines ou des boutiques de vente, etc. Il n'y a donc, croyons-nous, aucune raison sérieuse de supposer que les colibacilles qui souillent effectivement le lait (ainsi que nous l'avons dit et comme chacun peut le vérifier) puissent avoir une signification profondément différente de ceux qui souillent les eaux : leur présence $n$ 'est ni dans un cas ni dans l'autre, un indice certain de la présence du typhique ; mais dans le lait comme dans les eaux, elle signifie "souillure ", directe ou indirecte, par des germes dont l'origine intestinale, proche ou lointaine, 
n'est pas douteuse. Cette manière de voir n'implique pas que le danger de la typhoïde d'origine lactée soit fréquent. Les épidémies de fièvre typhoïde dont le lait puisse être rendu responsable sont fort rares; et d'ailleurs le consommateur porte presque toujours à l'ébullition le lait qu'il a acheté, ce qui le protège contre l'éventualité d'une infection accidentelle du lait par des germes pathogènes variés (1).

Il n'en est pas moins vrai que la présence d'une forte quantité de colibacilles dans un lait est, à notre avis, un indice de moins bon augure que la présence de petites ou de très petites quantités de ce même germe, surtout si la recherche, dans les deux cas, est faite avant que la prolifération spontanée ait pu en multiplier le nombre. C'est pourquoi, sans vouloir, peut-être, donner absolument au colibacille dans le lait la même signification que dans les eaux, nous estimons que sa recherche et sa numération approximative présentent un réel intérêt au point de vue de l'hygiène, contrairement à ce qui a été dit et écrit par certains auteurs.

VASSILEFF, d'ailleurs, s'exprime ainsi sur cette question :

"... Puisque le coli-bacille est un hôte constant du gros intestin de l'homme et des animaux domestiques, qu'il se trouve en abondance dans les excréments de celui-là et de ceux-ci, sa présence dans le lait décèle à coup sûr la souillure de cet aliment par des matières excrémentielles et, de plus, elle signifie la possibilité que le lait peut avoir véhiculé à un moment donné des germes pathogènes qui coexisteraient à côté du coli-bacille.

"Quand l'analyse de l'eau permet d'y trouver le bacille typhique, le $B$. coli accompagne toujours ce dernier; mais l'inverse n'est pas fatalement vrai.

«Une eau dans laquelle on décèle le $B$. coli est évidemment suspecte, mais elle ne contient pas obligatoirement le B. typhosus. A fortiori, une eau sans $B$. coli est également sans B. typhique.

"Ces considérations sont également d'application stricte au lait ; leur importance ne saurait échapper à l'hygiéniste soucieux de procurer du bon lait à l'enfance pour ses débuts.

"Aussi la question de la contamination du lait par les matières fécales est-elle du plus haut intérêt, car si le lait est souillé, il est toujours suspect, en attendant qu'il puisse être dangereux.

"La présence du coli-bacille dans le lait pasteurisé est gépéra-

(1) Notons en passant qu'il serait hautement préférable de consommer des laits dont la pureté baetériologique serait telle qu'il deviendrait inutile de les faire bouillir. Mais cette possibilité est exceptionnelle. Il faut disposer de laits extraordinairement soignés, comme celui auquel nous faisions allusion plus haut, pour qu'un médecin puisse en recommander l'usage sans ébullition préalable. 
lement considérée comme une indication de chauffage insuffisant ou de réinfection après la pasteurisation.

"Ainsi, le lait pasteurisé du commerce peut-il présenter des dangers analogues à ceux du lait cru, attendu que si dans celui-ci les ferments lactiques inoffensifs empêchent les ferments nuisibles, dans le lait pasteurisé les ferments lactiques sont en partie tués en raison de la température à laquelle le lait est porté... »)

2. Raisons d'ordre industriel. - A ces raisons s'en ajoutent d'autres d'ordre industriel.

En effet :

Quel contrôle avons-nous de l'efficacité des pasteurisations, de la désinfection des appareils, de l'asepsie des opérations ? Les industriels avertis font faire des numérations microbiennes en cours de fabrication. Cela suffit-il ? N'oublions pas que les pasteurisations (hautes et rapides ou basses et prolongées) laissent des germes dans le lait : thermophiles, sporogènes̃, etc. En été ces germes sont, dans les laits, comme dans la nature, plus abondants qu'en hiver. Certaines circonstances fortuites, à la production, peuvent en augmenter notablement le nombre (influence de la nature des fourrages, de la conduite de la traite, ete.). Done, à égalité de soins et d'habituelle efficacité des pasteurisations, celles-ci laissent inévitablement passer des quantités de germes qui peuvent être très variées et dont le nombre et la nature peuvent dépendre exclusivement de la production.

Comment faire la discrimination entre ces circonstances (auxquelles l'industriel ne peut rien en général, puisqu'elles lui sont étrangères) et celles qui proviendraient de la marche défectueuse d'un appareil ? Certes, on pourrait le savoir en procédant à une analyse bactériologique complète du lait pasteurisé. Mais dans le contrôle quotidien d'une usine, cet examen complet est impossible.

La numération, à elle seule, malgré son extrême utilité, ne suffit done pas. Le problème qui se pose est, en effet, de savoir si le mode de pasteurisation adopté détruit bien, tous les jours, tous les germes qu'il doit exterminer. Le contrôle de la qualité du travail et du bon fonctionnement des appareils, devrait donc toujours se faire par la recherche dans le lait pasteurisé d'un germe que la pasteurisation doit normalement détruire. Il faudrait évidemment que ce germe existât en permanence et en abondance dans le lait cru qui arrive à l'usine et que la recherche en fût facile, rapide et non ambiguë. Or, le colibacille remplit toutes ces conditions. Toujours présent dans les laits qui viennent de l'étable, il doit être toujours complètement exterminé dans le lait pasteurisé pris aseptiquement au sortir des 
appareils. Si on le retrouve dans le lait prélevé dans ces conditions, on fait, du même coup, la preuve d'une défectuosité dans la pasteurisation. C'est pourquoi la recherche du coli dans le lait pasteurisé est un élément de contrôle autrement précieux et significatif qu'une simple numération de germes. Nous osons écrire que cette recherehe est primordiale et qu'elle justifie pleinement par conséquent (en dehors des raisons relevant de l'hygiène), l'étude que nous avons entreprise.

\section{§ 3. - DE LA SENSIBILITÉ A EXIGER DE LA RECHERCHE} DU COLIBACILLE DANS LE LAIT.

Quand un laboratoire est consulté en vue de la recherche du colibacille dans un échantillon de lait, la question lui est souvent posée sous la forme suivante : Ce lait contient-il ou ne contient-il pas de B. Coli? Poser la question de cette façon, constitue, à notre avis, une erreur.

En effet :

Le $B$. Coli est, avons-nous dit, très répandu dans les laits courants. II s'y rencontre, en quantités il est vrai fort variables, mais d'une manière régulière et constante. Respecter la question à la lettre et y répondre dans le même sens, exposerait, pour peu que l'analyse soit toujours effectuée sur quelques centimètres cubes de lait, à donner dans tous les cas une réponse affirmative dont l'interprétation serait impossible. Ce qui importe surtout, répétons-le, ce n'est pas de savoir si le lait examiné contient du colibacille, mais de connaître le nombre approximatif de germes de $B$. Coli qu'il renferme. Or, la fixation d'un mode opératoire rationnel, la détermination des limites de sensibilité à donner à la méthode de recherche, sont fonction de la valeur qu'on attache à la présence de telle ou telle quantité de coli dans le lait.

Il s'agit en somme ici de discuter non plus de la signification qualitative du coli dans le lait, comme nous l'avons fait précédemment, mais d'essayer d'interpréter maintenant sa signification quantitative. Là aussi l'hygiéniste et l'industriel doivent avoir leur mot à dire.

10 Point de vue de I'hygiène. - Il semble évident, à priori, en raison de ce que nous avons exposé plus haut, que, du point de vue de l'hygiène, un lait sera d'autant meilleur qu'il contiendra moins de colibacilles. Mais à quelles limites s'arrêter? Le même problème s'est posé pour l'eau et une sorte d'accord tacite s'est fait autour des chiffres proposés par VINCENT.

Un résultat identique pourra-t-il être acquis pour le lait ? Il semble, à priori, que la chose soit impossible; car le lait est un milieu de culture de choix ; et tel lait déclaré bon le matin, risque- 
rait d'être rejeté le soir, alors qu'au strict point de vue de l'hygiène, s'il ne contenait au départ aucun germe pathogène, il pourrait encore être considéré comme excellent.

Pourra-t-on, comme on l'a fait d'ailleurs aussi pour l'eau, lier la question du nombre de colibacilles à celle du nombre de germes totaux ? La question est extrêmement délicate, car la richesse du lait en germes totaux, à n'importe quel moment, dépend beaucoup du nombre de germes laissés par la pasteurisation ou introduits accidentellement après. Tel lait dont la teneur en germes élevée pourrait faire croire à une multiplication spontanée des germes initiaux - de nature à atténuer par conséquent la signification du nombre des colibacilles - peut être beaucoup plus récent, mais avoir été plus infecté que tel autre, plus ancien, mais moins souillé à l'origine et done moins riche en germes totaux. Nous pourrions en citer maints exemples.

D'ailleurs en admettant même que cette liaison «nombre de germes totaux-nombre de coli " fût possible, l'atténuation de la portée du coli dans un lait, du fait de son enrichissement par pullulation spontanée, encourrait le grave reproche de ne pas tenir compte de ce que cette possibilité de multiplication du coli peut s'appliquer aussi bien à des germes pathogènes (tel que le typhique) s'il s'en était introduit dans le lait à l'origine.

Si, done, aucune liaison ne paraît possible entre ces deux données, mieux vaudrait semble-t-il, risquer de pécher par excès de prudence et attribuer, dans tous les cas, au colibacille une signification proportionnelle au nombre de ses représentants, sans tenir compte du nombre total des autres germes. On échapperait ainsi à la critique précédente, qui est sérieuse, et le problème se simplifierait grandement. On arriverait finalement à établir une échelle d'ordre de grandeurs basée sur la pratique, comme on l'a fait pour les eaux. Il resterait à savoir, bien entendu, comment on devrait chiffrer cette échelle et quelle interprétation il faudrait lui donner. En un tel domaine, la parole est plus à l'hygiéniste qu'au bactériologiste. Malheureusement cette donnée nous manque, cette échelle est fort difficile, sinon impossible à établir. Et cependant elle serait fort utile, non seulement pour l'interprétation de certaines analyses, mais encore pour obtenir l'unité de vues qui fait absolument défaut dans ce domaine et aussi, enfin, pour régler et simplifier les techniques analytiques. Quel congrès inscrira cette question à son ordre du jour et proposera des normes raisonnables ?

Il n'est ni dans notre compétence ni dans notre désir d'essayer de faire une proposition. Toutefois comme cette échelle - si elle voit jamais le jour - devra être fondée sur la pratique, nous croyons 
pouvoir rappeler quelques données expérimentales susceptibles de fixer tant soit peu les idées :

$1^{\circ}$ Une eau contenant plus de 100 coli au litre commence à être considérée comme suspecte.

$2^{\circ}$ En se montrant 10 fois moins sévère pour le lait, on tombe dans les teneurs minima des laits de vente courante à Paris, dont la grande majorité, pourtant, ne sauraient être considérés comme suspects, au point de vue de la signification du colibacille.

$3^{\circ}$ Notons toutefois que cette dernière affirmation peut être atténuée par l'habitude du consommateur de faire, chaque jour, bouillir son lait.

$4^{\circ}$ Enfin certains laits parviennent à réaliser des teneurs en colibacilles extraordinairement basses (moins de 20 au litre), voire complètement nulles sur des échantillons maxima soumis en entier à l'analyse.

On voit combien sera difficile à résoudre la question de la fixation de ees normes sous le rapport de l'hygiène. On pourrait nous répondre, et éluder ainsi le problème, en prétendant que sa solution est inutile. Il nous semble évident cependant qu'un lait fortement chargé en colibacilles est, quoi qu'on fasse, plus suspect qu'un lait qui n'en renferme que de petites quantités. Et cela, non pas tant par la présence du coli lui-même - auquel, malǵré tout, le typhique n'est que rarement associé - que par l'indice certain de la contamination qu'il révèle, laquelle pourrait prendre un jour, mais trop tard cette fois, une dangereuse signification.

Le moins qu'on puisse faire, en tout eas, si la question ne peut recevoir de meilleure réponse, serait de remplacer ces "degrés de suspicion " par des "degrés de qualité "; on définirait des laits de "grades " différents, en considérant non seulement le nombre de germes totaux mais aussi le nombre de colibacilles et en faisant de ces conditions des exigences simultanément nécessaires. A titre documentaire nous voudrions donner quelques exemples qui, s'ils n'engagent en rien l'avenir de l'idée, ont au moins le mérite d'être basés sur des analyses quotidiennes sérieuses.

La mise en vente à Paris d'un lait pasteurisé à basse température et renfermant ordinairement moins de 500 germes vivants au centimètre cube et moins de 50 colibacilles au litre (1), est un fait acquis (lait $\mathrm{OFCO}$ ). Comme ce lait est le plus pur que nous ayons jamais rencontré dans le commerce, parmi les laits pasteurisés, qu'il donne sur l'enfant des résultats cliniques extraordinaires - comme il fallait $s^{\prime} y$ attendre - et que son existence est définitivement possible et prouvée, nous serions fondés à prévoir un " premier grade " ayant ces caractéristiques.

(1) C'est-à-dire : absence de colibacilles sur des prises d'essai allant jusqu'à $20 \mathrm{~cm}^{3}$ de lait. 
Il existe aussi dans le commerce (mais en très petit nombre) des laits dont la qualité bactériologique, inférieure à celle du précédent, est néanmoins supérieure à celle de la moyenne des laits de grosse vente courante ; ces laits se caractérisent par des teneurs en germes comprises entre quelques dizaines de mille et quelques centaines de mille au centimètre cube, avec de 100 à 1.000 colibacilles au litre. On pourrait se représenter ces chiffres comme caractérisant un "deuxième grade".

Enfin la majorité de la production de la grosse industrie laitière en raison des difficultés énormes rencontrées tant à la récolte qu'au t traitement et au transport de grosses quantités de lait, et malgré les efforts inouïs accomplis en vue d'un progrès constant, et d'ailleurs réel, se caractérise par des nombres de germes totaux variant entre quelques centaines de mille et quelques millions et des teneurs en colibacilles très notablement supérieures à 1.000 au litre. Ces laits pourraient constituer le " troisième grade ".

La classe des laits suspects (si elle pouvait être définie) aurait des chances de se placer après les normes du troisième grade, sous réserve, peut-être, de préciser celles-ci davantage.

Empressons-nous d'ajouter qu'il faudrait fixer des normes d'été et des normes d'hiver, préciser et unifier les méthodes d'analyse, surtout la composition des milieux de numération (1) et le mode de recherche du colibacille, en vue d'unifier les principes de l'interprétation.

En un mot, qu'il s'agisse de fixer des "grades" de qualité, ou des normes d'interprétation de la "bactériométrie " et de la "colimétrie " des laits, nous pensons, qu'un jour ou l'autre, les hygiénistes auront à s'inquiéter de la signification qualitative et quantitative du colibacille dans le lait. Et cette idée ne doit pas laisser le bactériologiste indifférent; car les méthodes d'analyse qu'il aura à appliquer seront, dans une certaine mesure, conditionnées par les exigences de l'interprétation.

Pour nous, qui nous sommes proposés l'étude de la recherche du colibacille dans le lait, la connaissance de ces ordres de grandeur était loin d'être inutile.

En effet :

Certains auteurs effectuent cette recherche sur 1/20 de centimètre cube ; d'autres, sur $1 / 2$ ou $1 \mathrm{~cm}^{3}$. Les conclusions de ces recherehes sont évidemment très différentes entre elles et surtout très différentes de ce qu'elles seraient en opérant sur de plus grosses quantités. La recherche du coli sur $1 / 20$ de centimètre cube limite la numération

(1) Les chiffres donnés plus haut proviennent tous de numérations effectuées sur milieud'Orla-Jensen. Pour le cali, ils ont été obtenus en appliquant la technique que nous exposerons dans cette étude. 
à 20.000 au litre. La recherche sur $1 \mathrm{~cm}^{3}$ la limite à 1.000 au litre. Pour les raisons exposées précédemment, il semble done qu'il y ait intérêt dans de nombreux cas, à viser une plus grande sensibilité et à opérer sur de plus grands volumes de lait.

Telle est la conclusion analytique qui se dégage du souci de l'interprétation quantitative du coli au point de vue de l'hygiène.

$2^{\circ}$ Point de vue industriel. - Au point de vue industriel, et pour des raisons toutes différentes, la sensibilité de la recherche du coli doit être poussée plus loin encore. L'analyse du lait, pour l'industriel, constitue surtout un contrôle de fabrication. Nous avons dit plus haut quel était l'intérêt de la recherche du coli au sortir de certains appareils (de pasteurisation en particulier) comme moyen de contrôle de leur fonctionnement. Il est évident que cet examen sera d'autant plus sévère, et d'autant plus instructif, que la recherche portera sur un plus grand volume de lait. Bien entendu, il faudra également se fixer une limite dans ce domaine ; car on peut toujours supposer, si on n'a pas rencontré de coli sur $50 \mathrm{~cm}^{3}$, qu'on pourrait en rencontrer sur 1 litre ; que si sur un litre on n'en trouve point, on en découvrirait peut-être sur 10 litres... Mais cette exagération n'a pas de sens ; car, à la suivre, il n'y aurait plus de bactériologie possible. Il faut se fixer des limites raisonnables, aussi larges que possible d'ailleurs. La grande habitude que nous avons acquise des contrôles quotidiens du colibacille dans les appareils de laiterie les plus divers, nous a appris que si on ne rencontre pas de coli sur $50 \mathrm{~cm}^{3}$ de lait, on a fort bien travaillé, et, en fait, il est inutile d'aller plus loin. Il ne faut oublier que les germes rares ne sont pas également disséminés dans une masse de liquide, et à vouloir chercher une inutile perfection, on risque de se heurter à des difficultés analytiques pratiquement insurmontables, puisqu'il deviendrait nécessaire, pour des conclusions honnêtes, d'opérer sur de nombreux essais parallèles en faisant intervenir le calcul des probabilités. Cette petite critique pourrait nous être retournée quand nous parlons de prendre 20 ou $50 \mathrm{~cm}^{3}$ de lait. L'expérience nous a montré qu'en faisant des analyses sur $10,20 \ldots, 50 \mathrm{~cm}^{3}$ de lait on obtient des renseignements en général assez concordants, et en tout cas toujours très précieux pour le contrôle des pasteurisations et de l'asepsie des autres opérations.'

La conclusion pratique qui se dégage de cette discussion est la suivante :

Quelles que soient, dans l'avenir, les bases de l'interprétation quantitative du colibacille dans le lait, la recherche de ce microbe ne doit pas se limiter à $1 \mathrm{~cm}^{3}$ de lait, et encore moins à des fractions de centimètre cube. Il faut pouvoir atteindre de plus grandes sen- 
sibilités, soit qu'on se place au point de vue de l'hygiène, soit surtout que cette recherche ait des fins industrielles. Il faut, selon nous, pouvoir effectuer des recherches sur tous les volumes de lait compris entre 1 et $50 \mathrm{~cm}^{3}$, exceptionnellement plus. Or, si la recherche est classique et aisée sur $1 \mathrm{~cm}^{3}$, il n'en est pas de même sur de plus grands volumes.

C'est précisément cette question de la recherche du coli sur de grands volumes de lait que nous aurons surtout en vue dans l'étude qui va suivre et dont nous croyons avoir suffisamment démontré l'intérêt.

\title{
BIBLIOGRAPHIE.
}

[1] Th. VAssiLefF. Recherche et numération du coli-bacille dans le lait par la méthode du rouge neutre. Le Lait, avril 1932, 322.

[2] Axzers et Johnson. Journal of Agricultural Research, III (15 février 1915), 401-410.

[3] Tanner et Dubois Granville. Journal of Dairy Science, VIII (janvier 1925), 47.

[4] Johnson. The Milk dealer, 1926, 106.

[5] TANNER et WINDSOR. Journal of Dairy Science, mai 1929, 202.

[6] Dopter et Sacquépíe. Précis de bactériologie, 1321. (Baillière, 1927.)

[7] Dínent et Etrillard. Sur la recherche du B. Coli dans les eaux. Annales de l'Institut Pasteur, mars 1931, 277.

(A suivre.)

\section{L'INFECTION LATENTE DE LA MAMELLE ET SES RÉVEILS. LES MOYENS DE LA DÉPISTER}

\author{
par CH. PORCHER
}

Dosteur ès Sciences physiques.

(Suite.)

A QUOI SONT DUS LES FLOCONS DANS L'ÉPREUVE A L'ALIZAROL SUR DES LAITS ALCALINS ? - Si l'on alcalinise un lait normal avec de la soude ou de l'ammoniaque pour le plonger si peu que ce soit par son $p H$ dans la zone des laits alcalins, l'addition d'alcool à $70^{\circ}$ ne produit pas de précipité. Or, l'emploi du réactif de Morres détermine sur les laits de rétention et sur les laits malades, laits alcalins comme on le sait, des précipités. Rapidement, le mélange se divise en deux couches de teintes différentes : la couche inférieure faite de flocons plus ou moins grossiers et la couche supérieure, d'un liquide plus ou moins limpide, mais dont la teinte semble indiquer toujours un $p H$ plus marqué que celle du précipité qu'il surmonte,

A $p H$ égal, alcalin ou virant vers l'alcalinité, un lait normal alcalinisé et un lait malade, dit alcalin, réagissent donc différemment vis-à-vis du réactif de Morres en ne considérant la réaction que du côté du précipité. 